\title{
Análise de um circuito de corrente constante através dos conceitos da eletrostática
}

\author{
Analysis of a direct current circuit using electrostatic concepts \\ Anderson Fonseca ${ }^{1}$, Germano Maioli Penello ${ }^{* 10}$ \\ ${ }^{1}$ Universidade Federal do Rio de Janeiro, Instituto de Física, Rio de Janeiro, RJ, Brasil.
}

Recebido em 29 de outubro de 2021. Aceito em 29 de dezembro de 2021.

\begin{abstract}
A eletrostática e os circuitos de corrente constante são tradicionalmente discutidos sem que sua conexão seja claramente evidenciada. A estrutura conceitual e os métodos operacionais analíticos desenvolvidos no estudo da eletrostática não são utilizados na análise dos circuitos. Para promover a aproximação entre esses dois campos de estudo, será examinado um circuito composto por uma bateria e fios condutores onde a distribuição superficial de carga assume o protagonismo da relação causal quando se investiga o surgimento da corrente elétrica. Dessa forma, o conhecimento sobre a interação das cargas elétricas pode ser empregado para compreender os processos físicos que ocorrem nos componentes do circuito à medida que as conexões entre eles são estabelecidas. Ao se examinar o circuito dessa maneira, amplia-se o espectro de problemas que podem ser discutidos dentro do mesmo conteúdo.
\end{abstract}

Palavras-chave: eletrostática, circuito elétrico, carga superficial.

Electrostatics and constant current circuits are traditionally discussed without their connection being clearly evident. The conceptual framework and analytical operational methods developed in the study of electrostatics are not used in circuit analysis. In order to bring these two fields of study closer together, a circuit consisting of a battery and conducting wires will be examined where the surface distribution of charge takes center stage in the causal relationship when investigating the emergence of electric current. This allows the use of knowledge about the interaction of electric charges to be employed in the understanding of the physical processes occurring in the circuit components as the connections are established. By examining the circuit in this fashion, the spectrum of problems that can be discussed within the same content is broadened.

Keywords: electrostatics, electrical circuit, surface charge.

\section{Introdução}

A análise dos circuitos de corrente constante geralmente não aborda uma discussão sobre o campo eletromagnético. A compreensão da dinâmica do circuito é obtida simplesmente aplicando-se as leis de $\mathrm{Ohm}$ e de Kirchhoff. Uma das dificuldades encontradas para se levar adiante uma investigação do campo eletromagnético em um circuito de corrente constante se encontra na determinação da origem desse campo ou, mais especificamente, em se determinar a fonte do campo elétrico que provoca e orienta a corrente elétrica ao longo dos fios condutores do circuitt].

Os trabalhos de G. Kirchhoff [4] e W. Weber $2^{2}$ no século XIX indicavam que sobre a superfície de condutores

\footnotetext{
*Endereço de correspondência: gpenello@if.ufrj.br

1 Equivocadamente, pode-se pensar que a origem desse campo elétrico está na bateria [1. Os trabalhos de Assis e Hernandes 2 e A. J. Fonseca [3] apresentam algumas situações cuja análise pode ser suficiente para dissipar essa ideia e revelar uma pista de onde procurar as cargas elétricas que produzem o campo elétrico no interior dos fios.

${ }^{2}$ Uma discussão dos trabalhos de Weber e Kirchhoff pode ser encontrada em [2].
}

transportando correntes constantes havia uma distribuição superficial de carga. Ao longo da primeira metade do século XX, C. Schaefer ${ }^{3}$ A. Marcus [6] e A. Sommerfeld [7] demostraram que o campo elétrico no interior de um fio retilíneo percorrido por uma corrente constante é criado pelas cargas elétricas distribuídas na superfície do condutor.

No estado estacionário de corrente, o potencial elétrico em um segmento de um fio condutor ôhmico retilíneo, que se encontra distante das extremidades do fio, é obtido através da solução da equação de Laplace de forma análoga a das distribuições estáticas de carga 8 . Conhecida a função potencial, pode-se determinar a densidade superficial de carga no condutor e o campo elétrico criado por ela.

Apesar da existência de uma densidade de carga na superfície dos condutores percorridos por correntes

\footnotetext{
${ }^{3}$ De acordo com Varney e Fisher [5], C. Schaefer apresentou em 1932, a análise do caso de um fio retilíneo longo transportando corrente constante e sua solução mostrava a distribuição da carga, do campo e do potencial elétricos no fio. O resultado de Schaefer foi publicado no terceiro volume da sua coleção Einführung in die Theoretische Physik.
} 
constantes ser capaz de explicar as características do campo elétrico nos diferentes pontos do circuito, possibilitando que uma análise do campo eletromagnético e da sua interação com a matéria seja realizada, constatase que o aparato conceitual desenvolvido durante o estudo dos fenômenos eletrostáticos permanece distante da metodologia de análise dos circuitos elétricos de corrente constante.

Inspirada nos trabalhos de H. Härtel [9, 10], R. Chabay e B. Sherwood [11 e I. M. Sefton [12, a proposta aqui apresentada para análise de circuitos elétricos se baseia na distribuição superficial de carga, permitindo conciliar os conceitos estudados em eletrostática com a eletrodinâmica dos circuitos de corrente constante. Nossa intenção é demonstrar as potencialidades de uma análise que explora a relação causal/efetiva de uma interpretação microscópica, para a explicação dos fenômenos relacionados com o funcionamento de um circuito elétrico. Implicitamente, essa proposta também encoraja uma reavaliação do enfoque que geralmente é dado ao estudo dos problemas eletrostáticos e à análise dos componentes do circuito. Na eletrostática, as distribuições de carga, campo e potencial elétricos recebem um tratamento desvinculado dos processos dinâmicos em curso nos circuitos, embora estejam diretamente correlacionados. Ao se considerar o comportamento microscópico, a distribuição superficial de carga assume o papel principal na explicação dos fenômenos. Como identificou Jackson [13], existem três funções subordinadas à densidade superficial de carga: (i) manter o potencial elétrico ao longo do circuito; (ii) criar o campo elétrico no espaço exterior ${ }^{4}$ aos condutores; (iii) e garantir o fluxo confinado da corrente. Consequentemente, os fios do circuito elétrico não podem ser interpretados apenas como um caminho condutor que interliga os demais componentes, necessitando-se analisar, como nos outros integrantes, sua densidade superficial de carga.

Para descrever os detalhes dessa análise, elegemos um circuito composto apenas por fios condutores resistivos e uma bateria. Ainda que considerada demasiadamente simples, essa composição permite exemplificar e demonstrar o potencial do estudo proposto.

O comportamento dos componentes e do sistema formado por eles será examinado em cada etapa de construção do circuit ${ }^{5}$ Devido ao detalhamento abstrato, a análise do resultado das conexões entre os componentes

\footnotetext{
4 As cargas distribuídas na superfície dos condutores criam campo elétrico tanto interna quanto externamente ao condutor. Jackson [13] enfatiza apenas a existência do campo externo como forma de contradizer a convicção, historicamente difundida, de que o campo elétrico externo a um condutor percorrido por corrente constante era inexistente.

${ }^{5}$ É importante ressaltar que esse tipo de circuito se propõe a uma análise conceitual e não deve ser construído experimentalmente Conectar os dois terminais de uma fonte de tensão (seja uma pilha ou bateria) através de um fio, pode resultar em superaquecimento da fonte e do fio, podendo ocasionar queimaduras, lesões ou danos aos componentes do circuito.
}

será acompanhada por imagens que possibilitem descrevê-los com maior clareza.

\section{Análise dos componentes do circuito}

A investigação das características dos componentes do circuito permite que se estabeleçam os fundamentos do modelo interpretativo que será aplicado para compreender o que acontecerá quando as conexões entre eles forem realizadas, além do papel que desempenham durante o funcionamento do circuito.

\subsection{A bateria}

A bateria normalmente é identificada como um tipo de fonte portátil de energia. Além disso, esse dispositivo consegue manter constante a densidade de carga elétrica nos seus contatos (terminais ou polos) e, por isso, desempenha a função de sustentar a distribuição das cargas elétricas superficiais nos diferentes pontos do circuito.

A complexidade dos processos químicos que ocorrem no interior de uma bateria (ou, a compreensão do trabalho interno realizado pelas forças não-conservativas na bateria), pode ser simplificada utilizando-se a analogia com uma "bateria mecânica" (Figura 1), como a apresentada no livro-texto de R. Chabay e B. Sherwood [1]: um motor move uma esteira que retira elétrons de uma placa, deixando-a positiva e os transporta até a outra placa, tornando-a negativa. À medida que o processo de transferência de carga se desenvolve, torna-se cada vez mais difícil remover elétrons de um contato e depositálos sobre o outro por causa das forças de atração e repulsão existentes entre os elétrons e os terminais da bateria, que agora se encontram carregados. O acréscimo de cargas em cada terminal atinge o limite quando a força interna da pilha (que provoca a separação das cargas) se equilibra com as forças atrativas e repulsivas.

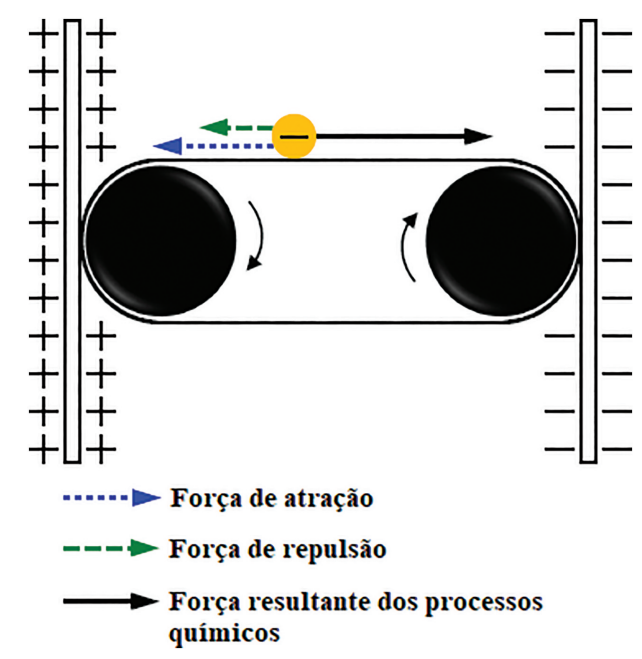

Figura 1: Modelo de uma "bateria mecânica". 
Esse processo mantém constante a densidade de carga em cada terminal e envolve o dispêndio de energia para o motor funcionar. De forma análoga, as forças que realizam o trabalho de transportar os elétrons de um polo ao outro (contrariando a ação das forças coulombianas) consomem a energia liberada nas reações químicas. Desse modo, o mecanismo de separação de carga (e sua sustentação em cada terminal enquanto o circuito estiver em funcionamento) pode ser discutido dentro do âmbito do formalismo utilizado na análise dos fenômenos eletrostáticos.

\subsection{O fio condutor}

Consideramos um fio condutor como um objeto metálico cuja estrutura é descrita conforme o modelo de condução elétrica apresentado em 1900 pelo físico alemão Paul Drude [14. Supõe-se que, quando os átomos de um metal formam suas ligações, os elétrons da camada de valência se separam dos átomos originais e passam a transitar livremente pelo volume do objeto. Dessa forma, um fio condutor é visualizado como uma rede de íons positivos estacionários permeada por um "mar" de elétrons que se deslocam desordenadamente através da rede com velocidade média $\langle\vec{v}\rangle$ nula, comportando-se como um gás de partículas livres. As interações eletromagnéticas tipo elétron-elétron e elétron-íon são desprezadas e somente as colisões mecânicas das partículas do gás (elétrons) com os íons da rede são consideradas. Esses eventos são tratados como instantâneos e ocorrem com uma probabilidade por unidade de tempo dada por $1 / \tau$, onde $\tau$ é um parâmetro fenomenológico denominado tempo de relaxação, considerado independente da posição e da velocidade do elétron. Devido às colisões, os elétrons são espalhados em todas as direções, percorrendo em seguida trajetórias retilíneas com uma energia cinética média que depende da temperatura absoluta local $T$, em acordo com a teoria cinética dos gases: $\frac{m}{2}\left\langle\vec{v}^{2}\right\rangle=\frac{3}{2} k T$. Os parâmetros $m$ e $k$ são, respectivamente, a massa do elétron e a constante de Boltzmann.

O comportamento exibido pela estrutura do condutor (como descrita pelo modelo de Drude) quando submetida a campos elétricos externos, possibilita investigar com maiores detalhes o mecanismo da condução elétrica.

Sob a ação de um campo elétrico $\vec{E}$, tanto os íons da rede como os elétrons de condução ficam sujeitos à força $\vec{F}=q \vec{E}$, onde $q$ é a carga do elétron ou do íon. A ação do campo desloca o mar de elétrons, enquanto os íons permanecem estacionários. Portanto, durante um breve intervalo de tempo (transiente), até que o equilíbrio eletrostático seja atingido, o movimento aleatório dos elétrons livres é ligeiramente alterado pela ação do campo, fazendo com que sua velocidade média deixe de ser nula e estabelecendo no condutor um deslocamento orientado dos elétrons móveis. Nesse sentido, a classificação do material como condutor ou isolante encontra-se vinculada à análise da resposta do material à ação de um campo elétrico.
De acordo com a interpretação clássica da condução elétrica, os íons da rede atuam como obstáculos ao movimento dos elétrons de condução. O fluxo dos elétrons através do condutor é amortecido por causa das colisões com os íons da rede. O efeito dissipativo produzido pelas colisões é interpretado macroscopicamente como a resistência elétrica do condutor. Microscopicamente, as informações cinemáticas associadas à dinâmica dessas colisões estão reunidas na resistividade elétrica do material. Essa grandeza está, portanto, relacionada à resistência elétrica. Nos condutores ôhmicos, a resistividade é independente do campo elétrico.

Durante as colisões uma parcela da energia (que originalmente se encontrava na bateria) é transmitida à rede de íons, provocando o aumento da sua agitação térmica e resultando no aquecimento do condutor. O fluxo de energia da bateria para o condutor acarreta o aumento da energia cinética dos elétrons de condução que é dissipada nas colisões.

\section{Conectando os fios condutores aos contatos da bateria}

Examinar as consequências da conexão de um fio condutor neutro com o terminal de uma bateria não difere muito de se analisar a ligação do mesmo fio com um objeto condutor eletrizado. Conforme é mostrado na Figura 2, quando a conexão é estabelecida com o terminal positivo da bateria, elétrons são transferidos do fio condutor para o terminal, dando origem a um fluxo transitório de carga. Esse deslocamento dos elétrons de condução em direção ao terminal positivo da bateria ocorre devido a ação do campo elétrico $\vec{E}$ criado pelas cargas distribuídas no termina $]^{6}$ Como resultado, a densidade de íons positivos do fio gradativamente tornase maior que a dos elétrons de condução. No equilíbrio, os elétrons de condução serão tão fortemente atraídos pela rede de íons quanto pelo terminal da bateria, interrompendo o fluxo e fazendo com que os elétrons livres retornem ao estado de velocidade média nula. De modo similar, o contato do fio com o terminal negativo faz com que os elétrons livres sejam repelidos e mais elétrons sejam adicionados ao fio, deixando-o com um excesso de carga negativa. Essa transferência de elétrons do terminal da bateria para o fio continua até que ocorra o equilíbrio entre a repulsão causada pelos elétrons do terminal e aquela provocada pelo excesso de carga que foi adicionado ao fio. A densidade de carga dos terminais da bateria não diminui como aconteceria caso a conexão fosse estabelecida com um objeto condutor eletrizado. À medida que esse processo se desenvolve, o trabalho realizado pelas forças não-conservativas existentes no interior da bateria mantém constante a densidade de carga sobre o terminal, conforme foi descrito na seção 2.1

\footnotetext{
${ }^{6} \mathrm{O}$ deslocamento dos elétrons de condução já acontece durante a aproximação do fio condutor e o terminal da bateria.
} 


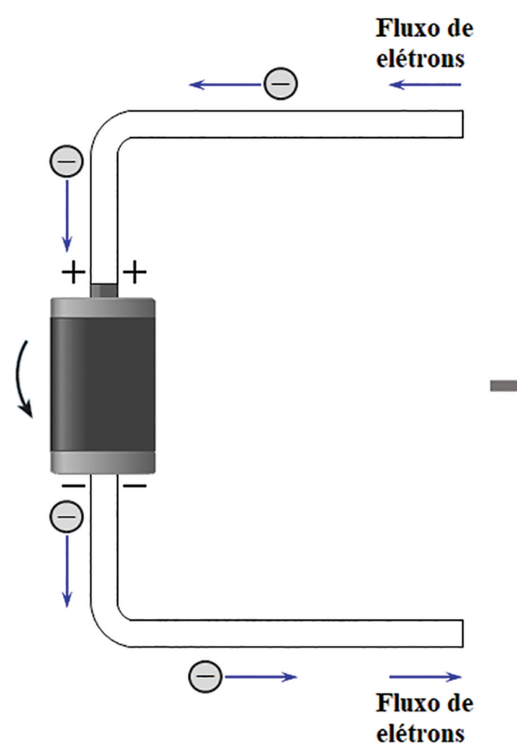

(a) Movimento dos elétrons de condução logo após a conexão dos fios (transiente).

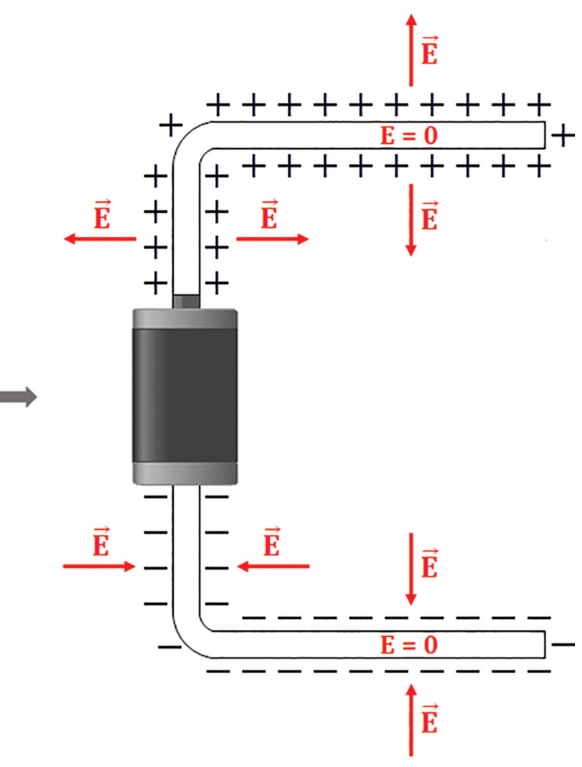

(b) Estado eletrostático alcançado instantes após a conexão dos fios.

Figura 2: Estados transiente e estático durante a conexão dos fios com a bateria. A forma como as cargas se distribuem nas torções ou extremidades do fio não está sendo explorada.

No equilíbrio eletrostático, a carga excedente estará distribuída na superfície dos fios e sua densidade será igual à dos terminais da bateria 7 Os fios, portanto, transformam-se em prolongamentos dos terminais. Se o fio for cilíndrico, à exceção das curvas e das extremidades, a distribuição de carga será uniforme ao longo do condutor. O campo elétrico no interior dos fios será nulo, porém imediatamente fora deles será diferente de zero e perpendicular à superfície. A existência de componentes do campo elétrico não-nulas, internas ou tangenciais à superfície, viola a condição de equilíbrio eletrostático.

A análise da conexão do fio condutor com o terminal da bateria também permite discutir qualitativamente outra quantidade física associada às distribuições de carga e introduzir a abordagem mais comumente utilizada para investigar a situação. O surgimento de um fluxo de elétrons nos condutores possibilita ampliar a discussão do fenômeno e examinar o trabalho $W_{a \rightarrow b}$ realizado sobre uma porção definida de carga $q$ quando ela é deslocada entre os pontos $a$ e $b$ pelo campo elétrico $\vec{E}$, o que nos remete, por conseguinte, ao consumo de uma quantidade da energia $U$ associada ao sistema formado pela carga deslocada e pelas cargas distribuídas no terminal: $W_{a \rightarrow b}=-\left(U_{b}-U_{a}\right)$ [15. O trabalho realizado pela força elétrica por unidade de carga deslocada será, portanto,

$$
\frac{W_{a \rightarrow b}}{q}=-\left(\frac{U_{b}}{q}-\frac{U_{a}}{q}\right)=V_{a}-V_{b} .
$$

\footnotetext{
7 Considerando que os fios se diferenciem dos terminais apenas no comprimento e desprezando efeitos de pontas e curvaturas.
}

Com o resultado da equação (1) podemos dizer que o fluxo de elétrons livres entre o fio condutor e o terminal (ou simplesmente através do fio) é motivado pela existência de uma diferença na quantidade escalar $V$, denominada potencial elétrico. A função que determina o potencial elétrico depende das características da distribuição de carga que o produz e da distância entre ela e o ponto considerado. $\mathrm{O}$ potencial elétrico pode ser interpretado como a capacidade que uma distribuição de carga possui para deslocar uma porção definida de carga, sendo tal habilidade identificada quantitativamente como energia por unidade de carga. Dessa forma, a cada ponto do espaço ao redor de cada terminal da bateria se associam um vetor campo elétrico e um potencial elétrico.

Utilizando agora argumentos sobre o potencial elétrico, podemos substituir a análise realizada a partir da interação dos elétrons livres do fio com o campo elétrico criado pelo terminal para explicar o deslocamento de carga até o equilíbrio eletrostático. Seguindo o sentido da interação coulombiana, cargas negativas se deslocam da região de menor para a de maior potencial. Como o fio condutor encontra-se inicialmente neutro, podemos considerar que seu potencial é nulo. Ao ser conectado ao terminal positivo da bateria, devido à diferença de potencial existente entre eles, elétrons serão deslocados do fio para o terminal da bateria. À medida que o processo se desenvolve, o potencial elétrico do fio se torna cada vez mais positivo devido à remoção de carga negativa. O limite é alcançado quando o potencial elétrico do fio se iguala ao do terminal. Nesse momento, 
como não há mais diferença de potencial, o fluxo de elétrons para o terminal positivo cessa. O equilíbrio eletrostático é atingido, portanto, quando terminal e fio se encontram no mesmo potencial. De modo semelhante, o resultado da conexão entre o fio e o terminal negativo pode ser encontrado, devendo-se observar apenas que, como nesse caso elétrons serão deslocados do terminal da bateria para o fio, o potencial elétrico do fio se torna cada vez mais negativo até se igualar ao do terminal. Como se pode perceber, a análise da conexão entre o fio condutor e o terminal da bateria explora nada mais que o aparato conceitual que tipicamente é estudado em eletrostática.

\section{Sistema formado pelos condutores carregados}

O passo seguinte à conexão dos fios aos terminais da bateria é a união das extremidades livres dos condutores, acompanhada de uma exploração das consequências que esse procedimento acarreta no circuito. Antes, porém, é interessante observar que o sistema formado pelos fios condutores carregados (Figura 3 traz consigo a possibilidade de se discutir os aspectos relacionados ao capacitor, um dispositivo largamente encontrado em circuitos elétricos. A análise que vem sendo explorada ao longo desse trabalho oportuniza que esse componente seja estudado antes mesmo que o resistor seja introduzido no circuito.

Estendido para um sistema de condutores carregados com cargas elétricas $+Q$ e $-Q$, com potenciais $V_{(+)}$e $V_{(-)}$, o conceito de capacidade elétrica ou capacitância $C$ é definido como

$$
C=\frac{Q}{V_{(+)}-V_{(-)}}=\frac{Q}{V},
$$

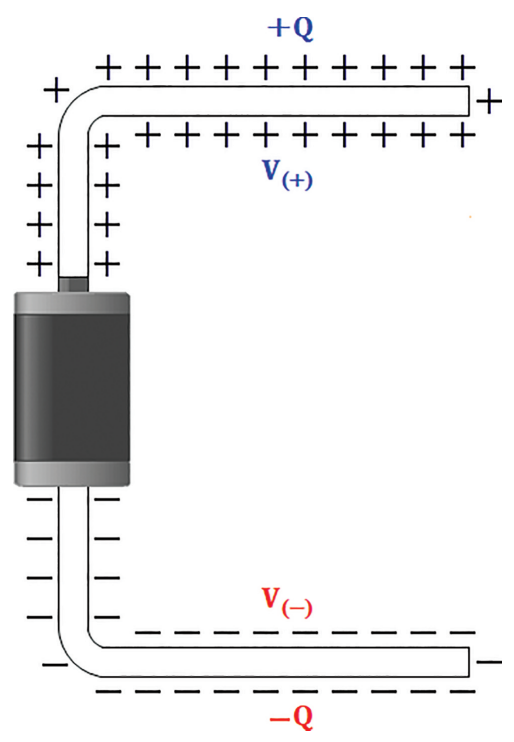

Figura 3: Sistema de dois condutores com cargas iguais, mas opostas. onde $V=V_{(+)}-V_{(-)}$é a diferença de potencial entre os condutores.

Apesar da capacitância estar presente em qualquer situação em que exista uma diferença de potencial entre elementos condutores, um capacitor é comumente analisado como um sistema constituído por duas placas condutoras planas e paralelas separadas por uma distância $d$, com o espaço entre elas podendo ser o vácuo ou preenchido por um dielétrico. Na situação ideal, considera-se que as placas são planos infinitos e o campo elétrico no intervalo entre as placas é uniforme em toda a sua extensão, sendo dado por $\vec{E}=\frac{\sigma}{\epsilon} \hat{n}$, onde $\sigma$ é a densidade superficial de carga da placa, $\epsilon$ é a permissividade elétrica do meio que existe entre as placas e $\hat{n}$ é o vetor unitário normal à placa. Obtém-se uma aproximação do caso idealizado fazendo com que a separação entre as placas de um capacitor real seja muito menor que as dimensões das placas. Dessa forma, o campo elétrico nas regiões próximas ao centro das placas é, com muito boa aproximação, uniforme.

Conforme é mostrado na Figura 44 quando as extremidades livres dos condutores estiverem próximas uma da outra o suficiente para satisfazer a condição $d \ll a$, sendo $a$ o diâmetro do fio, a região central do sistema formado por elas poderá ser considerada como um capacitor de placas planas e paralelas, o que possibilita a investigação dos conceitos físicos associados ao componente capacitivo. A forma como a aproximação das extremidades afeta a distribuição de carga ao longo dos fios, assim como o efeito produzido sobre ela pelas regiões pontiagudas dos fios não será discutida nesse trabalho. O leitor interessado encontrará em [16] um método semiquantitativo para determinação aproximada da densidade de carga em circuitos com diferentes geometrias.

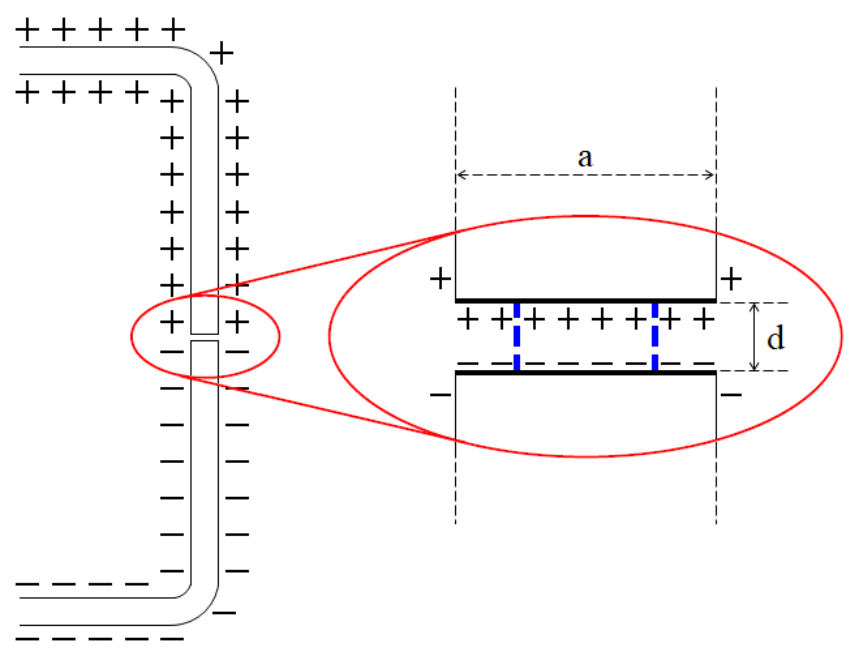

Figura 4: Aproximação das extremidades dos fios condutores analisado de maneira similar a um capacitor de placas planas e paralelas. No detalhe, a região onde o campo elétrico é aproximadamente uniforme se encontra entre as linhas verticais tracejadas azuis. 


\section{Unindo as extremidades livres dos condutores}

Antes de unirmos as extremidades livres dos fios da Figura 4, é importante analisar mais detalhadamente a distribuição de carga elétrica nessas regiões e o campo elétrico no interior do condutor em pontos próximos das extremidades quando ainda existe entre elas um vão, como ilustrado na Figura 5. A distribuição das cargas nas extremidades dos fios é bem mais complexa do que a forma como a estamos representando. Contudo, podemos explorar a distribuição apresentada para determinar o efeito produzido pelas cargas no interior dos fios sem prejudicar o resultado. Para não sobrecarregar a análise, a forma como as cargas se distribuem nas torções ou extremidades do fio não será explorada, assim como o efeito produzido na densidade superficial de carga provocado pela indução eletrostática 8

Como o sistema está em equilíbrio, o campo elétrico no interior dos condutores é nulo. Sendo assim, em qualquer ponto interno, os campos elétricos produzidos pelas cargas que se encontram nas paredes do vão e aqueles produzidos por todas as outras cargas da superfície do fio se equilibram (Figura 6).

Quando eliminamos o vão unindo as extremidades dos fios, as cargas distribuídas sobre as paredes neutralizam a região interna e o campo devido a elas deixa de

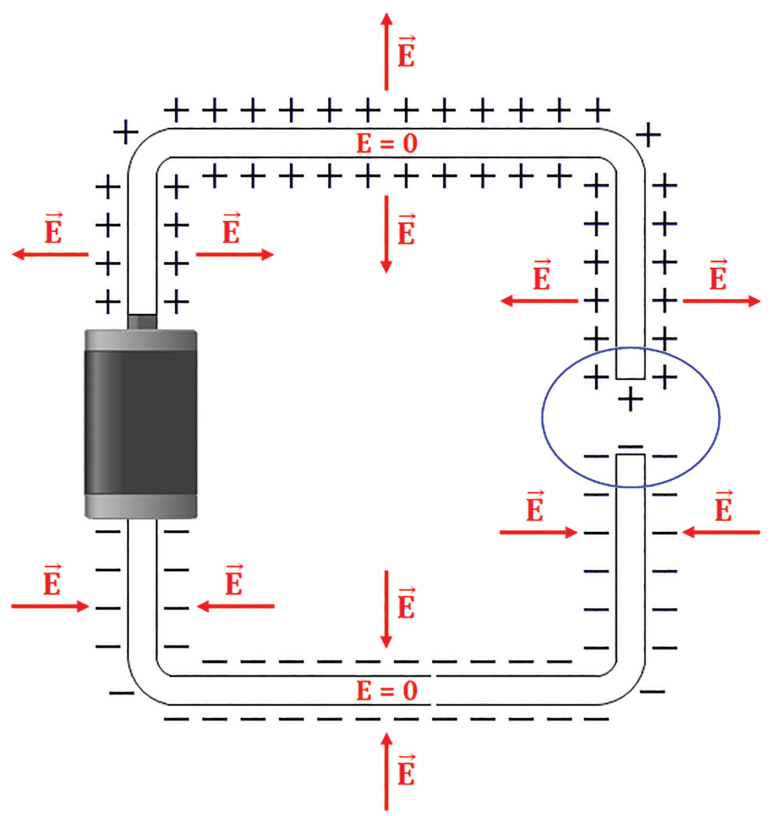

Figura 5: Fios condutores conectados à bateria em equilíbrio eletrostático. A região destacada será analisada antes de se realizar a conexão das extremidades.

\footnotetext{
8 A análise da variação da distribuição de carga sobre os fios à medida que suas extremidades são aproximadas pode ser feita considerando-se as faces das extremidades como um capacitor, cuja distância $d$ entre as placas varia, mas a diferença de potencial entre elas é mantida constante.
}

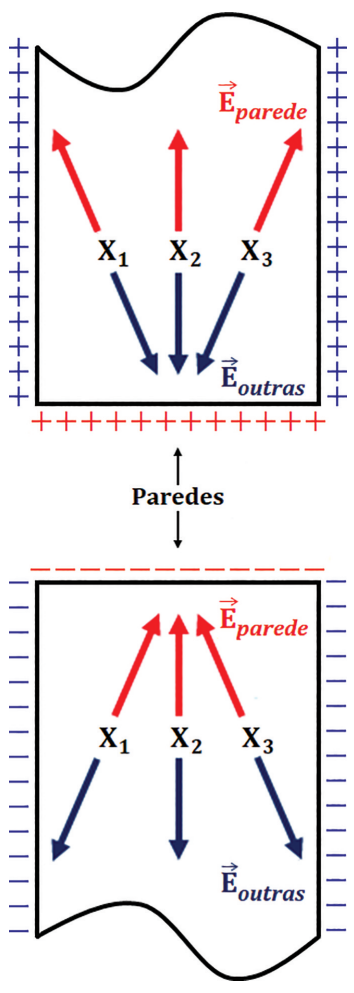

Figura 6: Campos produzidos pelas cargas das paredes do vão ( $\left.\vec{E}_{\text {parede }}\right)$ e de todas as outras cargas da superfície dos fios ( $\left.\vec{E}_{\text {outras }}\right)$ nos pontos $\mathbf{X}_{\mathbf{1}}, \mathbf{X}_{\mathbf{2}}$ e $\mathbf{X}_{\mathbf{3}}$.

existir, restando apenas os campos devido às outras cargas superficiais e uma descontinuidade instantânea na densidade superficial de carga na região onde ocorreu a conexão (Figura 7). Por causa do campo restante, os elétrons de condução serão colocados em movimento provocando a diluição da distribuição superficial de carga e eliminando a descontinuidade. $\mathrm{Na}$ porção superior da junção, elétrons livres serão deslocados para a superfície tornando menos positiva a densidade superficial de carga; na porção inferior, elétrons se afastarão da superfície deixando a distribuição superficial de carga mais positiva. Na Figura 8 é mostrada a distribuição superficial de carga decorrido um intervalo de tempo muito curto após a junção das extremidades, mas não o suficiente para que o estado estacionário tenha sido alcançado. A movimentação de portadores de carga livres já foi capaz de alterar a densidade superficial de carga se aproximando de uma variação gradual. Em nossa simbologia, quanto maior a quantidade de sinais + ou -, maior a densidade de carga.

As mudanças na densidade superficial de carga são induzidas pelo campo elétrico interior gerado pelas próprias cargas superficiais. À medida que a distribuição de carga é modificada, o campo elétrico no interior do condutor assume uma nova configuração e essas modificações se propagam com uma rapidez $v$ comparável à velocidade da luz no vácuo [17]. Isso quer dizer que em pontos distantes do local onde houve a conexão, as 


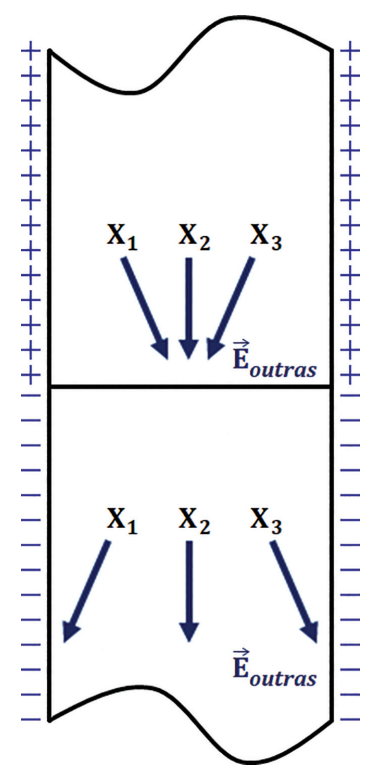

Figura 7: Campos produzidos apenas pelas cargas distribuídas na superfície dos fios imediatamente após a união.

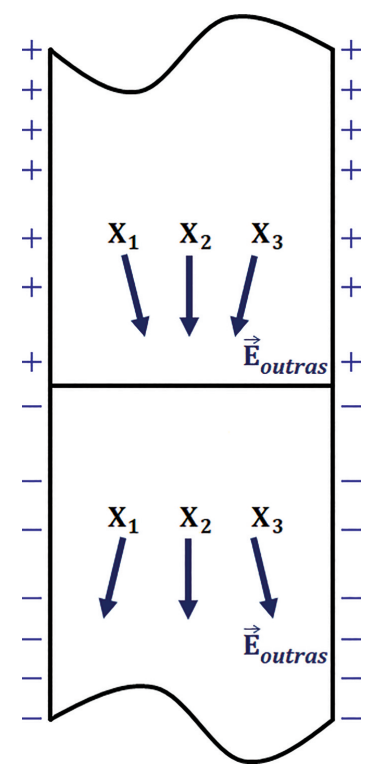

Figura 8: Densidade superficial de carga diluída pouco tempo depois que ocorre a junção das extremidades dos fios. O campo elétrico assume uma nova configuração devido à mudança na densidade superficial de cargas: à medida que o tempo passa a direção do campo elétrico ficará cada vez mais alinhada com as paredes do fio condutor; ao mesmo tempo, a densidade superficial de cargas passa a exibir cada vez mais uma dependência linear com o comprimento do fio.

cargas da superfície permanecem distribuídas conforme se encontravam durante o equilíbrio eletrostático até que a informação (o campo elétrico) de que o circuito se completou as alcance. O intervalo de tempo que o campo elétrico resultante da distribuição superficial de carga no fio demora para alcançar uma região do condutor a uma distância $d$ de onde o circuito se completou é dado por $\Delta t=d / v$. Logo, em pouquíssimo tempo a distribuição superficial de carga e, consequentemente, o campo elétrico deixam de variar com o tempo, significando que o estado estacionário foi alcançado. Combinando as equações de Maxwell com a lei de Ohm e a condição de corrente estacionária obtém-se um conjunto de equações que definem o fluxo de corrente em um meio de condutividade elétrica constante [18], a saber:

$$
\begin{aligned}
\vec{\nabla} \cdot \vec{J} & =0 \\
\vec{J} & =\sigma \vec{E} \\
\vec{\nabla} \times \vec{E} & =\overrightarrow{0},
\end{aligned}
$$

onde $\sigma$ é a condutividade elétrica do condutor e $\vec{J}$ é a densidade de corrente elétrica.

Decorre das equações (3) e (4) que $\vec{\nabla} \cdot \vec{E}=0$. Combinando esse resultado com a lei de Gauss $\vec{\nabla} \cdot \vec{E}=$ $\frac{\rho}{\epsilon_{0}}$ (onde $\rho$ é a densidade volumétrica de carga e $\epsilon_{0}$ é a permissividade elétrica do vácuo), conclui-se que o interior do condutor é neutro.

Paralelamente, como a equação $\sqrt{5}$ implica que $\vec{E}=$ $-\vec{\nabla} V$, e visto que $\vec{\nabla} \cdot \vec{E}=0$, constata-se que o potencial elétrico dentro do condutor precisa satisfazer a equação de Laplace, $\nabla^{2} V=0$.

Estas conclusões mostram que condutores ôhmicos transportando corrente constante, têm seu interior caracterizado por: $\sigma$ constante; $\rho=0 ; \nabla^{2} V=0$. Em razão disso, juntamente com argumentos de simetria, obtém-se que no interior de um fio condutor retilíneo infinitamente longo e de seção circular uniforme, constituído de material homogêneo e isotrópico, cercado por um meio isolante de permissividade elétrica constante, conduzindo corrente constante, o campo elétrico apresenta em todos os pontos a mesma componente ao longo da direção do eixo do condutor ${ }^{9}$. Consequentemente, o potencial elétrico deve ser uma função linear da distância medida ao longo da direção do eixo do condutor.

Fora do condutor, o potencial elétrico também precisa satisfazer a equação de Laplace, pois se considera que nas proximidades do fio não existem distribuições de carga e que os terminais da bateria ligados a ele estão muito distantes. Como as soluções interna e externa para o potencial devem respeitar as mesmas condições de contorno na superfície do condutor, a função que descreve o potencial externo deve também apresentar um termo com a mesma dependência em relação à distância medida ao longo da direção do eixo do condutor exibida pelo potencial interno. Por causa disso, a densidade superficial de carga varia linearmente em relação à distância medida ao longo da direção do eixo do condutor [7]. Devido à simetria da superfície do condutor em relação ao seu eixo, conclui-se que em fios condutores cilíndricos de seção circular, a densidade superficial de

\footnotetext{
9 Externamente, além da componente paralela ao eixo do condutor, existe uma componente perpendicular à superfície dele.
} 
carga é uma função linear da distância medida ao longo da direção do eixo do condutor ${ }^{10}$

Como demonstrou Russel [19], admitindo-se que a componente do campo elétrico na direção do eixo do condutor é constante, os resultados encontrados para o potencial elétrico e para densidade superficial de carga em condutores cilíndricos com seções circulares podem ser estendidos para condutores cilíndricos com seções transversais arbitrárias. Entretanto, deve-se notar que em condutores cilíndricos com seções diferentes da circular, o potencial e o campo elétrico fora do fio, e a densidade de carga superficial dependerão também da geometria da seção dos condutores. Os interessados em se familiarizar com os detalhes matemáticos envolvidos na solução do problema de se determinar a configuração do campo elétrico produzido por um fio resistivo ôhmico longo, retilíneo e de seção circular, com uma condutividade elétrica uniforme e percorrido por uma corrente elétrica de intensidade constante, a partir de uma densidade superficial de carga preestabelecida, poderão consultar os trabalhos de Coombes e Laue 20 e Assis et al [21. As Figuras 9 e 10 ilustram qualitativamente o resultado encontrado por Russell. O circuito completo está ilustrado na Figura 11 .

Embora a solução formal do problema de se determinar a origem do campo elétrico que provoca e orienta a corrente elétrica nos fios condutores fuja do escopo desse trabalho, é conveniente apresentar argumentos que possibilitem a compreensão da conclusão de que a fonte do campo se encontra, principalmente, nas cargas elétricas distribuídas na superfície do condutor e não na bateria, e que a direção do campo se mantém paralela ao eixo do condutor em toda a sua extensão. O resultado da configuração exibida pelo campo elétrico é o surgimento de uma corrente elétrica de intensidade constante percorrendo o fio e a bateria.

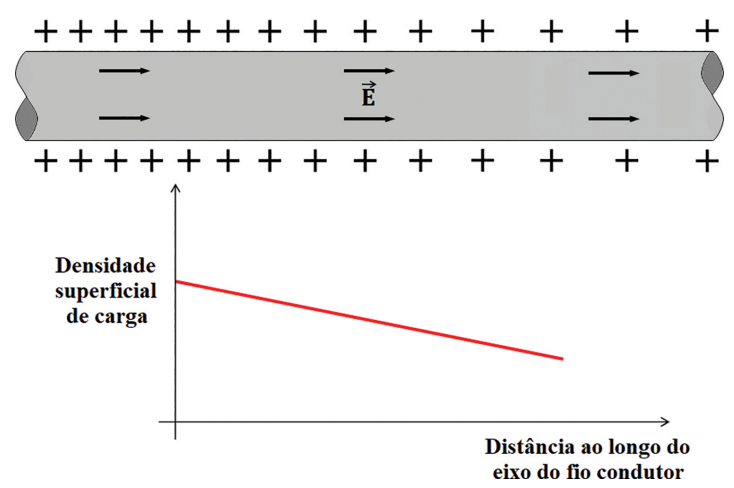

Figura 9: Comportamento da densidade superficial de carga em um segmento de um fio condutor ôhmico retilíneo e infinitamente longo no estado estacionário de corrente. Imagem extraída do trabalho de H. Härtel [10] (adaptada).

\footnotetext{
$\overline{10}$ As hipóteses assumidas são boas aproximações mesmo para circuitos realistas, desde que se esteja analisando pontos do fio longe de seus terminais e longe de qualquer região na qual o fio esteja curvado.
}

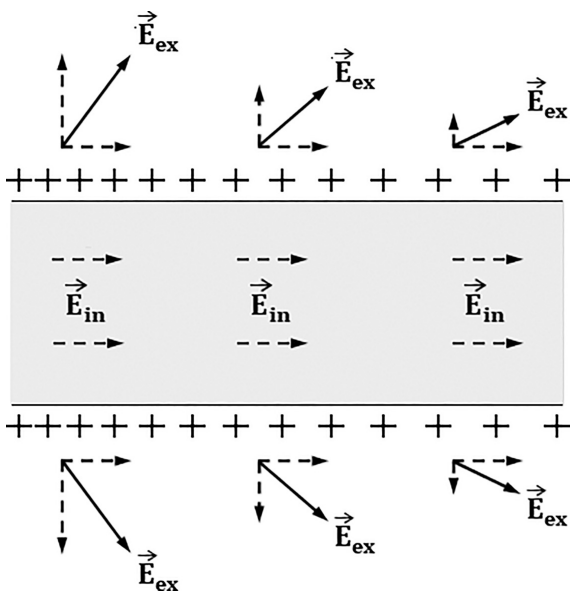

Figura 10: Distribuição de carga e campos elétricos interno $\left(\vec{E}_{i n}\right)$ e externo $\left(\vec{E}_{e x}\right)$ em um segmento de um fio cilíndrico conduzindo uma corrente constante. A porção considerada do fio encontra-se distante das extremidades do condutor.

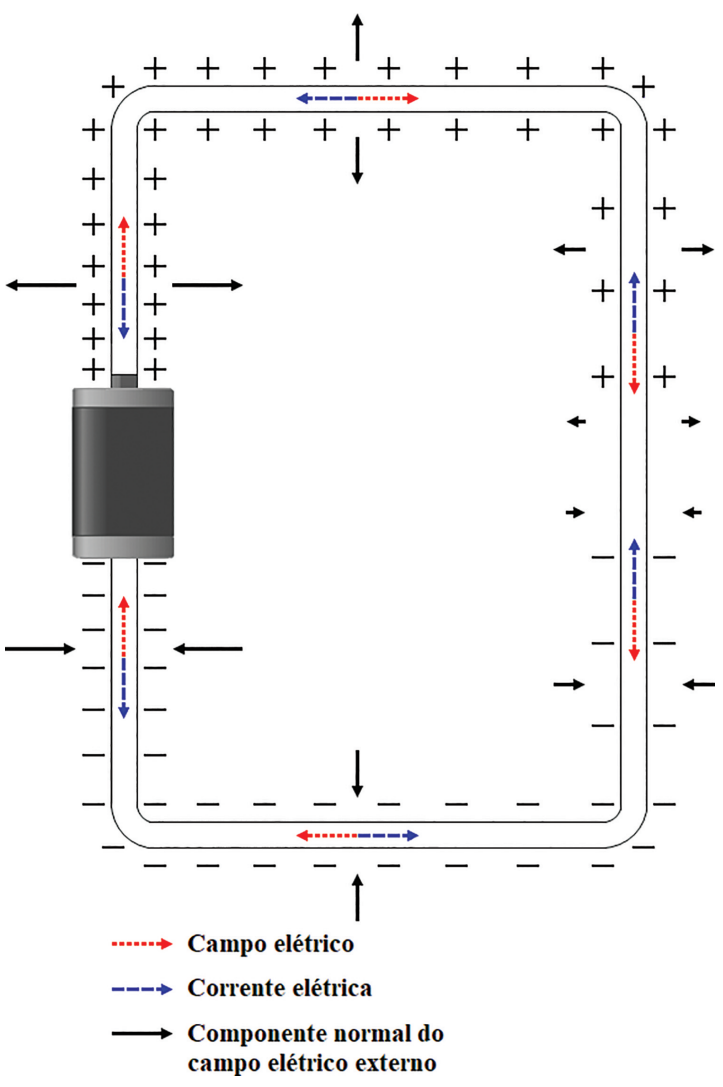

Figura 11: Campo elétrico, corrente elétrica e distribuição superficial de carga aproximada ao longo do circuito no regime estacionário. Apenas a componente normal do campo elétrico externo foi representada.

Uma possibilidade para abordar o problema da origem do campo diretor da corrente é considerar que o campo elétrico em um ponto qualquer do espaço, produzido por um fio condutor cilíndrico e retilíneo, cuja distribuição superficial de cargas varia linearmente com o 


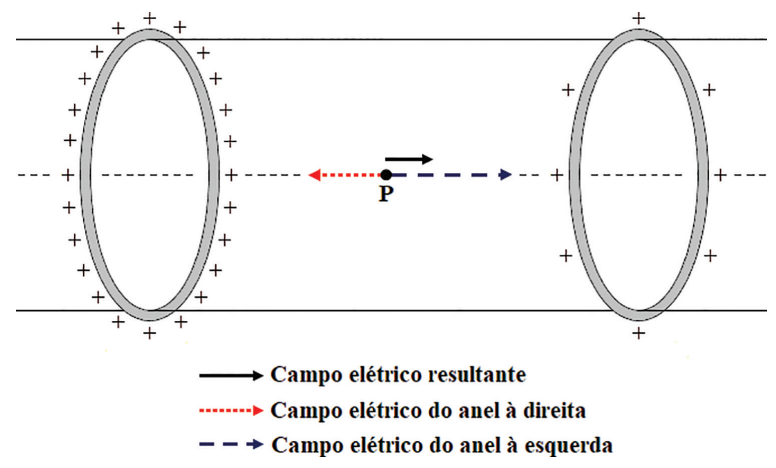

Figura 12: Campo elétrico resultante de dois anéis com densidade de carga elétrica superficial desigual.

seu comprimento, pode ser obtido pela superposição dos campos produzidos pela sucessão de anéis carregados de largura infinitesimal que compõem a carga superficial do fio. Valendo-se de argumentos de simetria, pode-se concluir que os campos produzidos pelos anéis em um ponto $\mathbf{P}$ do eixo do condutor são sempre orientados ao longo desse mesmo eixo. Tomando-se, então, dois anéis equidistantes do ponto considerado, haverá um campo elétrico resultante em $\mathbf{P}$ devido à desigualdade nas densidades de carga dos anéis, como pode ser observado na Figura 12. Outra alternativa consiste em explorar o comportamento da distribuição superficial de carga observado no regime transiente e no estado estacionário. É durante o transiente que a densidade superficial de carga é modificada. No interior do fio o campo elétrico apresenta direções transversais e paralelas ao eixo do condutor, conforme o ponto considerado (observe a Figura 8). Os campos transversais são responsáveis por remover elétrons da superfície ou adicioná-los a ela. Quando o estado estacionário é atingido, as variações na densidade superficial de carga são interrompidas, indicando que campos elétricos com direções transversais ao eixo não existem mais. Como o movimento dos elétrons de condução no estado estacionário é caracterizado por apresentar $\langle\vec{v}\rangle \neq 0$, porém constante, recorrendo ao modelo de Drude, pode-se concluir que o campo elétrico no interior do fio nessa condição, além de ser paralelo ao eixo do condutor, também possui módulo constante.

Embora tenhamos dirigido nossa atenção para os trechos retilíneos do fio, a configuração do campo elétrico derivada da análise das equações (3), (4) e (5), em associação com a lei de Gauss, pode ser estendida para as regiões curvas, mas a conclusão a respeito do gradiente da densidade superficial de carga não é verdadeira nessas regiões. Como o formato do fio não produz campo elétrico e o módulo do campo é o mesmo em todos os pontos do condutor, alguma modificação local tem que ser responsável pela mudança na orientação do campo elétrico. Logo, é conveniente analisar o que acontece nessas regiões, corroborando a necessidade de se discutir onde se encontra a fonte do campo elétrico que provoca a corrente.

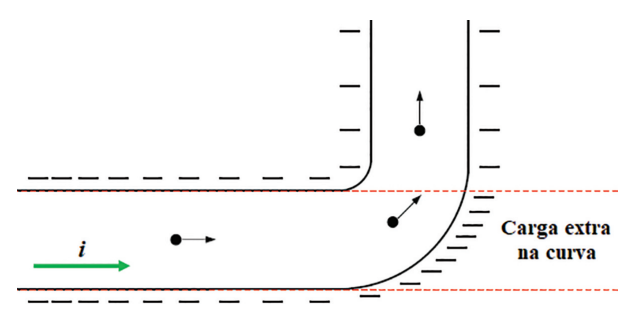

Figura 13: Processo de acúmulo de carga na curva de um fio condutor.

Nas regiões torcidas de um fio conduzindo uma corrente elétrica constante, o campo elétrico permanece com a mesma intensidade, mas sua direção se modifica, mantendo-se paralelo ao eixo do fio. O que explica a mudança de direção do campo elétrico é a modificação local na distribuição superficial de cargas elétricas nas redondezas do segmento curvado do fid ${ }^{11}$ O trabalho de Sefton [12] ajuda entender qualitativamente como se processa a mudança na densidade superficial de carga nessas regiões.

Os elétrons de condução precisam estar sujeitos à ação de uma força resultante fora da sua direção de movimento para que eles possam contornar a curva do fio. Caso contrário, os elétrons continuarão sua trajetória de aproximação terminando por acumularem-se na parte externa da curva. Cada porção de carga acumulada na curva contribui para o aumento da força que desvia os outros elétrons que se aproximam. Esse processo cumulativo se encerra quando a região adquire carga superficial suficiente para desviar os elétrons o necessário para percorrerem a curva seguindo a direção do eixo do fio. A situação discutida está representada na Figura 13

Como se pode observar, o conhecimento adquirido acerca dos fenômenos eletrostáticos permite compreender o funcionamento de um circuito elétrico de corrente constante e explorar aspectos que vão além das possibilidades que o modelo de análise tradicionalmente aplicado pode alcançar.

\section{Conclusão}

A proposta de análise que foi apresentada aborda de forma qualitativa a interação do campo eletromagnético com a estrutura dos componentes de um circuito para interpretar fenômenos observados durante a sequência de construção do circuito e o comportamento do sistema quando em funcionamento. Aplicada a um circuito de corrente constante, a abordagem discutida permite que a análise empregada nas situações envolvendo cargas elétricas estáticas seja utilizada no estudo de um circuito constante.

Ao avaliar as interações microscópicas à medida que as conexões entre os componentes são estabelecidas, a

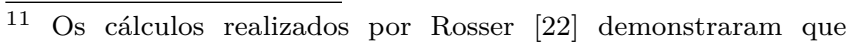
a configuração do campo elétrico é particularmente sensível a mudanças extremamente pequenas na distribuição das cargas. 
análise possibilita que o comportamento do circuito seja examinado fora do estado estacionário, algo não explorado no tratamento habitualmente aplicado aos circuitos de corrente constante. Durante as etapas de construção do circuito, o modelo de análise também oportuniza a discussão de assuntos muitas vezes explorados apenas no âmbito da eletrostática, como processos de eletrização e blindagem eletrostática.

O sistema formado pelos condutores carregados possibilita que se reconheça a abrangência do conceito de capacitância, podendo ser utilizado como ponto de partida para uma investigação sobre as variadas situações em que essa característica pode se manifestar, evidenciando importância de sua análise nos circuitos elétricos. Isso torna possível que o capacitor seja o primeiro dispositivo a ser apresentado em uma análise de circuitos, substituindo o protagonismo do resistor. Quando as extremidades dos fios são aproximadas para realizar a sua junção, tem-se a oportunidade de se investigar as características de um capacitor ideal, um elemento comumente encontrado na composição dos circuitos.

Por fim, a análise teórica desse circuito, composto apenas por um fio e uma bateria, ressalta que a fonte do campo elétrico que direciona a corrente no circuito se encontra nas cargas elétricas distribuídas na superfície do condutor e não na bateria. A direção do campo elétrico se mantém paralela ao eixo do condutor em toda a sua extensão no momento em que o estado estacionário é atingido. Esse campo elétrico é responsável por orientar a corrente elétrica de intensidade constante que percorre todo o circuito, evidenciando a correlação existente entre a eletrostática e a eletrodinâmica dos circuitos de corrente constante.

\section{Referências}

[1] R. Lynch, American Journal of Physics 51, 272 (1983).

[2] A.K.T. Assis e J.K. Hernandes, A força elétrica de uma corrente: Weber e as cargas superficiais de condutores resistivos com correntes constantes (Edusp, São Paulo, 2009), p. 205.

[3] A.J. Fonseca, A eletrostática oculta na eletrodinâmica dos circuitos elétricos de corrente constante. Dissertação de Mestrado, Universidade Federal do Rio de Janeiro, Rio de Janeiro (2020).

[4] G. Kirchhoff, The London, Edinburgh, and Dublin Philosophical Magazine and Journal of Science 37, 463 (1850).

[5] R.N. Varney e L.H. Fisher, American Journal of Physics 52, 1097 (1984).

[6] A. Marcus, American Journal of Physics 9, 225 (1941).

[7] A. Sommerfeld, Lectures on theoretical physics: electrodynamics (Academic Press, New York, 1952), p. 125.

[8] J.B. Neto, Teoria eletromagnética: parte clássica. (Editora Livraria da Física, São Paulo, 2015), p. 173.
[9] H. Härtel, A qualitative approach to electricity, Technical Report No. IRL87-0001, Inst. for Research on Learning, Palo Alto (1987), disponível em: http://ww w1.astrophysik.uni-kiel.de/ hhaertel/PUB/Quality-El ectricity.pdf

[10] H. Härtel, Caderno Brasileiro de Ensino de Física 29 , 1015 (2012).

[11] R.W. Chabay e B.A. Sherwood, Matter and interactions: electric and magnetic interactions (John Wiley \& Sons, Hoboken, 2015), v. 2, p. 716 .

[12] I.M. Sefton, in 9th Science Teachers' Workshop, Sydney, 2002 (Science Foundation for Physics, Sydney, 2002), disponível em: https://pdfs.semanticscholar.org/45f8/ 8e04e25cdcd59d79b8140ed6d2a856275ad4.pdf acessado em 8 de abril de 2018.

[13] J.D. Jackson, American Journal of Physics 64, 855 (1996).

[14] C.A.S. Pérez, Caderno Brasileiro de Ensino de Física, 17, 348 (2000).

[15] H.D. Young e R.A. Freedman, Física III, (Pearson Education do Brasil, São Paulo, 2009), v. 3, p. 72.

[16] R. Müller, American Journal of Physics 80, 782 (2012).

[17] W.G.V. Rosser, American Journal of Physics 31, 884 (1963).

[18] A. Zangwill, Modern Electrodynamics (Cambridge University Press, New York, 2012), p. 272.

[19] B.R. Russell, American Journal of Physics 36, 527 (1968).

[20] C.A. Coombes e H. Laue, American Journal of Physics 49, 450 (1981).

[21] A.K.T. Assis, W.A. Rodrigues e A.J. Mania, Foundations of Physics 29, 729 (1999).

[22] W.G.V. Rosser, American Journal of Physics 38, 265 (1970). 